\title{
Bound-State Solution of s-Wave Klein-Gordon Equation for Woods-Saxon Potential
}

\author{
Eser Olğar and Haydar Mutaf \\ Engineering of Physics Department, University of Gaziantep, Gaziantep, Turkey \\ Correspondence should be addressed to Eser Olğar; olgar@gantep.edu.tr
}

Received 25 March 2015; Revised 20 June 2015; Accepted 25 June 2015

Academic Editor: Yao-Zhong Zhang

Copyright (C) 2015 E. Olğar and H. Mutaf. This is an open access article distributed under the Creative Commons Attribution License, which permits unrestricted use, distribution, and reproduction in any medium, provided the original work is properly cited.

The bound-state solution of s-wave Klein-Gordon equation is calculated for Woods-Saxon potential by using the asymptotic iteration method (AIM). The energy eigenvalues and eigenfunctions are obtained for the required condition of bound-state solutions.

\section{Introduction}

In relativistic quantum mechanics, the Klein-Gordon (KG) equation has a wide range of applications. Recently, there have been many studies on KG equation with various types of potentials by using different methods to describe the corresponding relativistic physical systems, namely, asymptotic iteration method [1], formal variable separation method [2], supersymmetric quantum mechanics [3-7], algebraic method [8-13], and Nikiforov-Uvarov method [14]. Most of these studies have been considered for equal or pure scalar $S(x)$ and vector potentials $V(x)$ cases. In this study, to have a bound-state solution, we use the proposed transformation in [15] with parameters $\beta$ which adjust the relation between scalar and vector Woods-Saxon potentials. Woods-Saxon potential [16] is a short range nuclear potential and it is important in all branches of physics. The bound-state solution of KG equation for Woods-Saxon potential has been of a great interest in the last decades with different methods [17-21]. It has been solved with Nikiforov-Uvarov method by using the Pekeris approximation to the centrifugal potential for any 1 -states for constant mass $[22,23]$ and for effective mass $[24,25]$.

In this paper, we use an alternative method which is called the asymptotic iteration method (AIM) [26] for obtaining the whole spectra of Woods-Saxon potentials. AIM has been used in many physical systems to obtain the corresponding eigenvalues and eigenfunctions [26-31]. Still, there are a few applications on KG equation with AIM [32-34] with a general transformation between vector and scalar potentials. To obtain the corresponding potential the vector potential is chosen in exponential potential form. Therefore, the KG equation reduces to Schrödinger-like equation with WoodsSaxon potential.

The organization of this paper is as follows: Section 2 deals with the formalism of the one-dimensional KG equation with scalar potential greater than vector potential. A general description of AIM is outlined in Section 3. The solution of Woods-Saxon potential in KG equation is treated in the subsequent section by means of AIM. Finally, Section 5 is devoted to conclusions.

\section{Formalism of the Klein-Gordon Equation}

Generally, the s-wave Klein-Gordon equation with scalar potential $S(x)$ and vector potential $V(x)$ can be written as [9]

$$
\begin{aligned}
& \left\{\frac{d^{2}}{d x^{2}}+\frac{1}{\hbar^{2} c^{2}}\left[(E-V(x))^{2}-\left(m c^{2}+S(x)\right)^{2}\right]\right\} f(x) \\
& \quad=0
\end{aligned}
$$

where $E$ represents the energy and $m$ represents the mass of the particle. In this equation, the radial wave function is expressed as $R(x)=f(x) / x$. We know that when the relation between vector and scalar potentials $S(x) \geq V(x)$, 
it yields real bound-state solutions. In this work, we consider the relation between potentials in the condition for boundstate solution which was introduced in [15] as

$$
S(x)=V(x)(\beta-1), \quad \beta \geq 0,
$$

where $\beta$ parameter is arbitrary constant. When $\beta=0,1,2$, the scalar potential leads to case of $S(x)=-V(x), S(x)=0$ (purely vector potential), and $S(x)=V(x)$ (equal potentials), respectively. When $\beta>2$, we get the required condition for the case of $S(x)>V(x)$. Substituting (2) into (1) yields

$$
\left\{\frac{d^{2}}{d x^{2}}-\frac{1}{\hbar^{2} c^{2}}\left[V_{\mathrm{eff}}(x)-\left(E^{2}-m^{2}\right)\right]\right\} f(x)=0,
$$

where the effective potential is

$$
V_{\mathrm{eff}}=V(x)\left[2 E+2 c^{2} m(\beta-1)+V(x)(\beta-2) \beta\right] .
$$

Now, the final equation is the transformed KG equation in the condition of unequal vector and scalar potentials. The following section deals with the corresponding method to solve the KG equation.

\section{Asymptotic Iteration Method}

The AIM is proposed to solve the second-order differential equations and the details can be found in [26]. First, we consider the following second-order homogeneous differential equation of the form

$$
y^{\prime \prime}(x)=\lambda_{0} y^{\prime}(x)+s_{0} y(x),
$$

where $\lambda_{0}$ and $s_{0}$ are functions and $y^{\prime}(x)$ and $y^{\prime \prime}(x)$ denote derivatives of $y$ with respect to $x$. It is easy to show that $(n+$ 2)th derivative of the function $y(x)$ can be written as

$$
y^{(n+2)}(x)=\lambda_{n} y^{\prime}(x)+s_{n} y(x) .
$$

The functions $\lambda_{n}$ and $s_{n}$ are given by the recurrence relations

$$
\begin{aligned}
& \lambda_{n}=\lambda_{n-1}^{\prime}+s_{n-1}+\lambda_{n-1} \lambda_{0}, \\
& s_{n}=s_{n-1}^{\prime}+\lambda_{n-1} s_{0} .
\end{aligned}
$$

For sufficiently large $n$, the ratio of the functions satisfies the following equations:

$$
\frac{\lambda_{n}}{s_{n}}=\frac{\lambda_{n-1}}{s_{n-1}}=\alpha(x) ;
$$

then the solution of (5) can be written as [26]

$$
\begin{aligned}
y(x)= & \exp \left(-\int^{x} \alpha d t\right) \\
& \cdot\left[C_{1}+C_{2} \int^{x} \exp \left(\int^{s}\left(\lambda_{0}+2 \alpha\right) d t\right) d s\right] .
\end{aligned}
$$

In calculating the parameters in (7), for $n=0$, we take the initial conditions as $\lambda_{-1}=1$ and $s_{-1}=0$ [29] and $\Delta_{n}(x)=0$ for

$$
\Delta_{n}(x)=\lambda_{n}(x) s_{n-1}(x)-\lambda_{n-1}(x) s_{n}(x),
$$

where $\Delta_{n}(x)$ is the termination condition in (8).
If the second-order homogeneous linear differential equation can be written in the form of [27]

$$
\begin{aligned}
y^{\prime \prime}(x)= & 2\left(\frac{a x^{N+1}}{1-b x^{N+2}}-\frac{(m+1)}{x}\right) y^{\prime}(x) \\
& -\frac{w x^{N}}{1-b x^{N+2}}
\end{aligned}
$$

using the function generator of AIM, it has an exact solution for $y_{n}(x)$ [27] as

$$
\begin{aligned}
y_{n}(x)= & (-1)^{n} C_{1}(\sigma)_{n}(N+2)^{n}(\sigma)_{n} \\
& \cdot{ }_{2} F_{1}\left(-n, \rho+n ; \sigma ; b x^{N+2}\right)
\end{aligned}
$$

with the defined parameters $(\sigma)_{n}=\Gamma(\sigma+n) / \sigma, \quad \sigma=(2 m+$ $N+3) /(N+2)$, and $\rho=((2 m+1) b+2 a) /(N+2) b$.

\section{Solution for Woods-Saxon Potential}

Let us consider the Woods-Saxon vector potential

$$
V(x)=\frac{-V_{0}}{1+e^{(x-R) / \alpha}} \quad \text { and then } S(x)=\frac{V_{0}(1-\beta)}{1+e^{(x-R) / \alpha}} .
$$

After substituting these potentials into (3) and changing the variables $y=1 /\left(1+e^{(x-R) / \alpha}\right)$, we obtain the second-order differential equation

$$
\begin{aligned}
& \left\{\frac{d^{2}}{d y^{2}}+\frac{(1-2 y)}{y(1-y)} \frac{d}{d y}-\frac{1}{y^{2}(1-y)^{2}}[\xi+A+B]\right\} \\
& \cdot f(y)=0
\end{aligned}
$$

with the corresponding parameters

$$
\begin{aligned}
\xi^{2} & =-\left(\frac{E^{2}-m^{2} c^{4}}{\hbar^{2} c^{2}}\right), \\
A & =\left(\frac{2 E V_{0} \alpha^{2}+2(\beta-1) c^{2} m V_{0} \alpha^{2}}{\hbar^{2} c^{2}}\right), \\
B & =-\left(\frac{(\beta-2) \beta V_{0}^{2} \alpha^{2}}{\hbar^{2} c^{2}}\right) .
\end{aligned}
$$

As we expected, (13) is transformed to the suitable form to apply the AIM. Therefore, (14) should have a solution in the form of "normalized" wave functions

$$
f(y)=y^{\xi}(1-y)^{v} \chi(y)
$$

with $v=\sqrt{\xi+A+B}$. Substituting (16) into (14), one obtains

$$
\begin{aligned}
\chi^{\prime \prime}(y)= & {\left[\frac{2 \xi(y-1)+2(1+v) y-1}{y(1-y)}\right] \chi^{\prime}(y) } \\
& +\left[\frac{(\xi+v)(1+2 \xi)+A}{y(1-y)}\right] \chi(y) .
\end{aligned}
$$

Within the framework of procedures of AIM, the functions $\lambda_{0}(y)$ and $s_{0}(y)$ can be written by comparing (17) with (5). It can be seen that $\lambda_{0}(y)$ and $s_{0}(y)$ functions are obtained by using the termination relation in (10) as 


$$
\begin{aligned}
\lambda_{0} & =\frac{1-2 \xi(y-1)-2(v+1) y}{y(y-1)}, \\
s_{0} & =\frac{2+(A-3 v-6) y+\left(4 v^{2}+9 v-A+6\right) y^{2}+3 \xi(y-1)(-2+(3+2 v) y)+2 \xi^{2}\left(y^{2}-3 y+2\right)}{y^{2}(y-1)^{2}} .
\end{aligned}
$$

By substituting these functions into the quantization condition, we get

$$
\begin{array}{ll}
\frac{s_{0}}{\lambda_{0}}=\frac{s_{1}}{\lambda_{1}} \Longrightarrow \xi_{0}+\sqrt{\xi+A+B}=-\frac{1}{2}-\frac{1}{2} \sqrt{1+4 A}, & n=0, \\
\frac{s_{1}}{\lambda_{1}}=\frac{s_{2}}{\lambda_{2}} \Longrightarrow \xi_{1}+\sqrt{\xi+A+B}=-\frac{3}{2}-\frac{1}{2} \sqrt{1+4 A}, & n=1, \\
\frac{s_{1}}{\lambda_{1}}=\frac{s_{2}}{\lambda_{2}} \Longrightarrow \xi_{2}+\sqrt{\xi+A+B}=-\frac{5}{2}-\frac{1}{2} \sqrt{1+4 A}, & n=2,
\end{array}
$$

Generalizing the above expressions, it is possible to write the general formula of $\xi$ as

$$
\xi_{n}+\sqrt{\xi+A+B}=-\frac{(2 n+1)}{2}-\frac{1}{2} \sqrt{1+4 A} .
$$

Using the definitions of parameters $\xi$ and $\varepsilon$, it can be shown that

$$
\begin{aligned}
& E_{n}^{2}-m^{2} c^{4}=-\hbar^{2} c^{2}\left(-\frac{(2 n+1)}{2}\right. \\
& \left.-\frac{1}{2} \sqrt{1+4 \frac{2 E V_{0} \alpha^{2}+2(\beta-1) c^{2} m V_{0} \alpha^{2}}{\hbar^{2} c^{2}}}-v\right) .
\end{aligned}
$$

Rearranging this expression, we get a more explicit expression for eigenvalues

$$
\begin{aligned}
E_{n} & = \pm\left[m^{2} c^{4}-\hbar^{2} c^{2}\left(-\frac{(2 n+1)}{2}\right.\right. \\
& -\frac{1}{2} \sqrt{1+4 \frac{2 E V_{0} \alpha^{2}+2(\beta-1) c^{2} m V_{0} \alpha^{2}}{\hbar^{2} c^{2}}} \\
& -v)]^{1 / 2}
\end{aligned}
$$

which is exactly the same as with the eigenvalue equation obtained in [21] through a proper choice of parameters.

4.1. Calculation of Eigenfunction. To get the normalized wave function, we have to compare (17) with the differential equation defined in (11). After some algebraic calculations, we get the required parameters as

$$
\begin{aligned}
a & =\frac{2 v+1}{2}, \\
b & =1, \\
N & =-1, \\
m & =\frac{2 E-1}{2}, \\
\sigma & =2 \xi+1, \\
\rho & =2 \xi-2 v+1 .
\end{aligned}
$$

Thus, these parameters yield a solution in the form of

$$
\begin{aligned}
& \chi_{n}(y)=(-1)^{n} C_{2} \\
& \cdot \frac{\Gamma\left(2 \xi_{n}+n+1\right){ }_{2} F_{1}\left(-n, 2\left(\xi_{n}-v\right)+1+n ; 2 \xi_{n}+1 ; y\right)}{\Gamma\left(2 \xi_{n}+1\right)}
\end{aligned}
$$

with the Gamma function $\Gamma$ and the Gauss hypergeometric function ${ }_{2} F_{1}$. With the aid of (24), the corresponding radial function becomes

$$
\begin{aligned}
R_{n}(x)= & (-1)^{n} N_{n} x\left(y^{\xi}(1-y)^{v}\right) \\
& \cdot{ }_{2} F_{1}\left(-n, 2\left(\xi_{n}-v\right)+1+n ; 2 \xi_{n}+1 ; y\right),
\end{aligned}
$$

where the normalization constant is

$$
N_{n}=C_{2} \frac{\Gamma\left(2 \xi_{n}+n+1\right)}{\Gamma\left(2 \xi_{n}+1\right)} .
$$

Substituting the values of $y$ in (25), the total radial differential equation transforms to

$$
\begin{aligned}
& R_{n}(y)=(-1)^{n} N_{n} x\left(\frac{\left(e^{(x-R) / \alpha}\right)^{v}}{\left(1+e^{(x-R) / \alpha}\right)^{v+\xi}}\right) \\
& \cdot{ }_{2} F_{1}\left(-n, 2\left(\xi_{n}-v\right)\right. \\
& \left.+1+n ; 2 \xi_{n}+1 ; \frac{1}{1+e^{(x-R) / \alpha}}\right) .
\end{aligned}
$$

After rearranging the parameters, this result is exactly the same as with those of calculated for Woods-Saxon potentials in literature $[17-19,21]$. 


\section{Conclusion}

In this study, we have obtained the whole spectrum of Woods-Saxon potential for s-wave KG equation by using AIM method. During the calculation, we have used the adjusting parameter $\beta$ between scalar and vector potentials to obey the bound-state solution condition. The eigenvalues and eigenfunction are calculated directly and exactly by using the procedure of AIM in simple way.

\section{Conflict of Interests}

The authors declare that there is no conflict of interests regarding the publication of this paper.

\section{Acknowledgments}

The research was supported by the Research Fund of Gaziantep University (BAP) and the Scientific and Technological Research Council of Turkey (TUBİTAK).

\section{References}

[1] E. Olğar, R. Koç, and H. Tütüncüler, "Bound states of the swave equation with equal scalar and vector standard Eckart potential," Chinese Physics Letters, vol. 23, no. 3, pp. 539-541, 2006.

[2] C.-F. Hou, X.-D. Sun, Z.-X. Zhou, and Y. Li, "General formulas for radial matrix elements of isotropic harmonic oscillators," Acta Physica Sinica, vol. 48, no. 3, pp. 385-388, 1999.

[3] C. Y. Chen, C. L. Liu, F. L. Lu, and D. S. Sun, "Bound states of the Klein-Gordon equation with n-dimensional scalar and vector hydrogen atom-type potentials," Acta Physica Sinica, vol. 52, pp. 1579-1584, 2003.

[4] Y. He, Z. Q. Cao, and Q. S. Shen, "Bound-state spectra for supersymmetric quantum mechanics," Physics Letters A, vol. 326, no. 5-6, pp. 315-321, 2004.

[5] G. Chen, Z.-D. Chen, and Z.-M. Lou, "Exact bound state solutions of the $s$-wave Klein-Gordon equation with the generalized Hulthén potential," Physics Letters. A, vol. 331, no. 6, pp. 374-377, 2004.

[6] W.-C. Qiang, "Bound states of the Klein-Gordon and Dirac equations for potential $V(r)=A r^{-2}-B r^{-1}$," Chinese Physics, vol. 12, no. 10, pp. 1054-1058, 2003.

[7] L.-Z. Yi, Y.-F. Diao, J.-Y. Liu, and C.-S. Jia, "Bound states of the Klein-Gordon equation with vector and scalar Rosen-Morsetype potentials," Physics Letters. A, vol. 333, no. 3-4, pp. 212-217, 2004.

[8] C.-S. Jia, Y. Li, Y. Sun, J.-Y. Liu, and L.-T. Sun, "Bound states of the five-parameter exponential-type potential model," Physics Letters. A, vol. 311, no. 2-3, pp. 115-125, 2003.

[9] F. Domínguez-Adame, "Bound states of the Klein-Gordon equation with vector and scalar Hulthén-type potentials," Physics Letters A, vol. 136, no. 4-5, pp. 175-177, 1989.

[10] Z.-Q. Ma, S.-H. Dong, X.-Y. Gu, J. A. Yu, and M. LozadaCassou, "The Klein-Gordon equation with a coulomb plus scalar potential in d dimensions," International Journal of Modern Physics E, vol. 13, no. 3, pp. 597-610, 2004.

[11] C.-Y. Chen, D.-S. Sun, and F.-L. Lu, "Relativistic bound states of Coulomb potential plus a new ring-shaped potential," Acta Physica Sinica, vol. 55, no. 8, pp. 3875-3879, 2006.
[12] W.-C. Qiang and S.-H. Dong, "Analytical approximations to the l-wave solutions of the Klein-Gordon equation for a second Pöschl-Teller like potential," Physics Letters A, vol. 372, no. 2728, pp. 4789-4792, 2008.

[13] S. S. Dong, S. H. Dong, H. Bahlouli, and V. B. Bezerra, "Algebraic approach to the Klein-Gordon equation with hyperbolic Scarf potential," International Journal of Modern Physics E, vol. 20, pp. 55-61, 2011.

[14] S. M. Ikhdair and R. Sever, "Exact solution of the KleinGordon equation for the PT-symmetric generalized WoodsSaxon potential by the Nikiforov-Uvarov method," Annalen der Physik, vol. 16, no. 3, pp. 218-232, 2007.

[15] E. Olğar, "Exact solution of Klein-Gordon equation by asymptotic iteration method," Chinese Physics Letters, vol. 25, no. 6, pp. 1939-1942, 2008.

[16] R. D. Woods and D. S. Saxon, "Diffuse surface optical model for nucleon-nuclei scattering," Physical Review, vol. 95, no. 2, pp. 577-578, 1954.

[17] J. Y. Guo, X. Z. Fang, and F. X. Xu, "Solution of the relativistic Dirac-Woods-Saxon problem," Physical Review A, vol. 66, Article ID 062105, 3 pages, 2002.

[18] C. F. Hou, Z. X. Zhou, and Y. Li, "Bound states of the Klein-Gordon equation with vector and scalar Wood-Saxon potentials," Acta Physica Sinica, vol. 8, no. 8, pp. 561-564, 1999.

[19] G. Chen, "Bound states for Dirac equation with wood-saxon potential," Acta Physica Sinica, vol. 53, no. 3, pp. 680-683, 2004.

[20] V. M. Villalba and C. Rojas, "Bound states of the Klein-Gordon equation in the presence of short range potentials," International Journal of Modern Physics A, vol. 21, no. 2, pp. 313-325, 2006.

[21] G. Chen, Z.-D. Chen, and Z.-M. Lou, "Exact bound state solutions of the $s$-wave Klein-Gordon equation with the generalized Hulthén potential," Physics Letters A, vol. 331, no. 6, pp. 374-377, 2004.

[22] V. H. Badalov, H. I. Ahmadov, and S. V. Badalov, "Any 1state analytical solutions of the Klein-Gordon equation for the Woods-Saxon potential," International Journal of Modern Physics E, vol. 19, no. 7, pp. 1463-1475, 2010.

[23] A. Altuğ and S. Ramazan, "Approximate $l$-state solutions of a spin-0 particle for Woods-Saxon potential," International Journal of Modern Physics C, vol. 20, no. 4, pp. 651-665, 2009.

[24] A. Altuğ and R. Sever, "Approximate $\ell$-state solutions to the Klein-Gordon equation for modified Woods-Saxon potential with position dependent mass," International Journal of Modern Physics A, vol. 24, pp. 3985-3999, 2009.

[25] A. Altuğ and R. Sever, "Bound states of the Klein-Gordon equation for Woods-Saxon potential with position dependent mass," International Journal of Modern Physics C, vol. 19, no. 5, pp. 763-773, 2008.

[26] H. Çiftçi, R. L. Hall, and N. Saad, "Asymptotic iteration method for eigenvalue problems," Journal of Physics. A. Mathematical and General, vol. 36, no. 47, pp. 11807-11816, 2003.

[27] N. Saad, R. L. Hall, and H. Çiftçi, "Sextic anharmonic oscillators and orthogonal polynomials," Journal of Physics A: Mathematical and General, vol. 39, no. 26, pp. 8477-8486, 2006.

[28] T. Barakat, K. Abodayeh, and A. Mukheimer, "The asymptotic iteration method for the angular spheroidal eigenvalues," Journal of Physics A: Mathematical and General, vol. 38, no. 6, pp. 1299-1304, 2005.

[29] F. M. Fernández, "On an iteration method for eigenvalue problems," Journal of Physics A. Mathematical and General, vol. 37, no. 23, pp. 6173-6180, 2004. 
[30] I. Boztosun, M. Karakoc, F. Yasuk, and A. Durmus, "Asymptotic iteration method solutions to the relativistic Duffin-KemmerPetiau equation," Journal of Mathematical Physics, vol. 47, Article ID 062301, 2006.

[31] A. Soylu, O. Bayrak, and I. Boztosun, "The energy eigenvalues of the two dimensional hydrogen atom in a magnetic field," International Journal of Modern Physics E, vol. 15, no. 6, pp. 1263-1271, 2006.

[32] E. Olğar and H. Mutaf, "Asymptotic iteration method for energies of inversely linear potential with spatially dependent mass," Communications in Theoretical Physics, vol. 53, no. 6, pp. 1043-1045, 2010.

[33] E. Olgar, "An alternative method for calculating bound-state of energy eigenvalues of Klein-Gordon for Quasi-exactly solvable potentials," Chinese Physics Letters, vol. 26, no. 2, Article ID 020302, 2009.

[34] E. Olğar, R. Koç, and H. Tütüncüler, "The exact solution of the s-wave Klein-Gordon equation for the generalized Hulthén potential by the asymptotic iteration method," Physica Scripta, vol. 78, no. 1, Article ID 015011, 2008. 


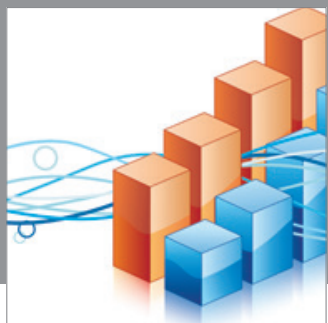

Advances in

Operations Research

mansans

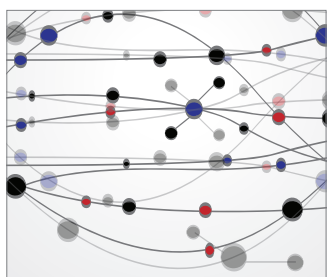

The Scientific World Journal
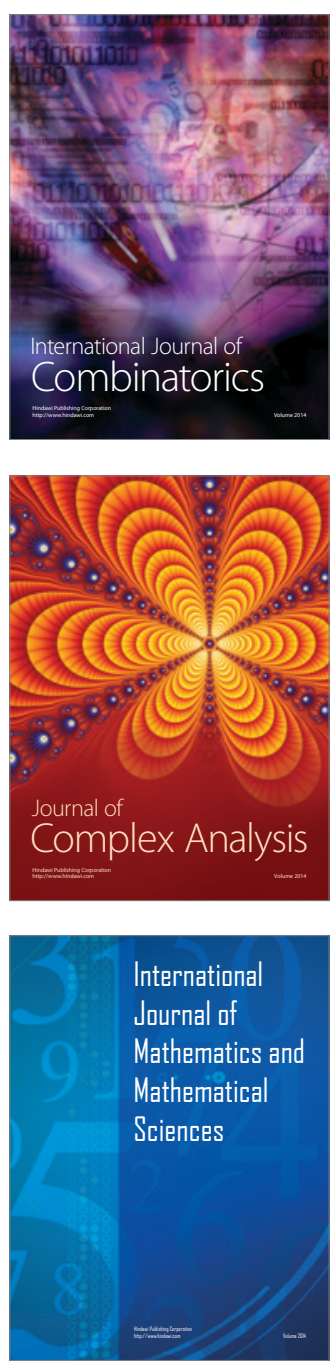
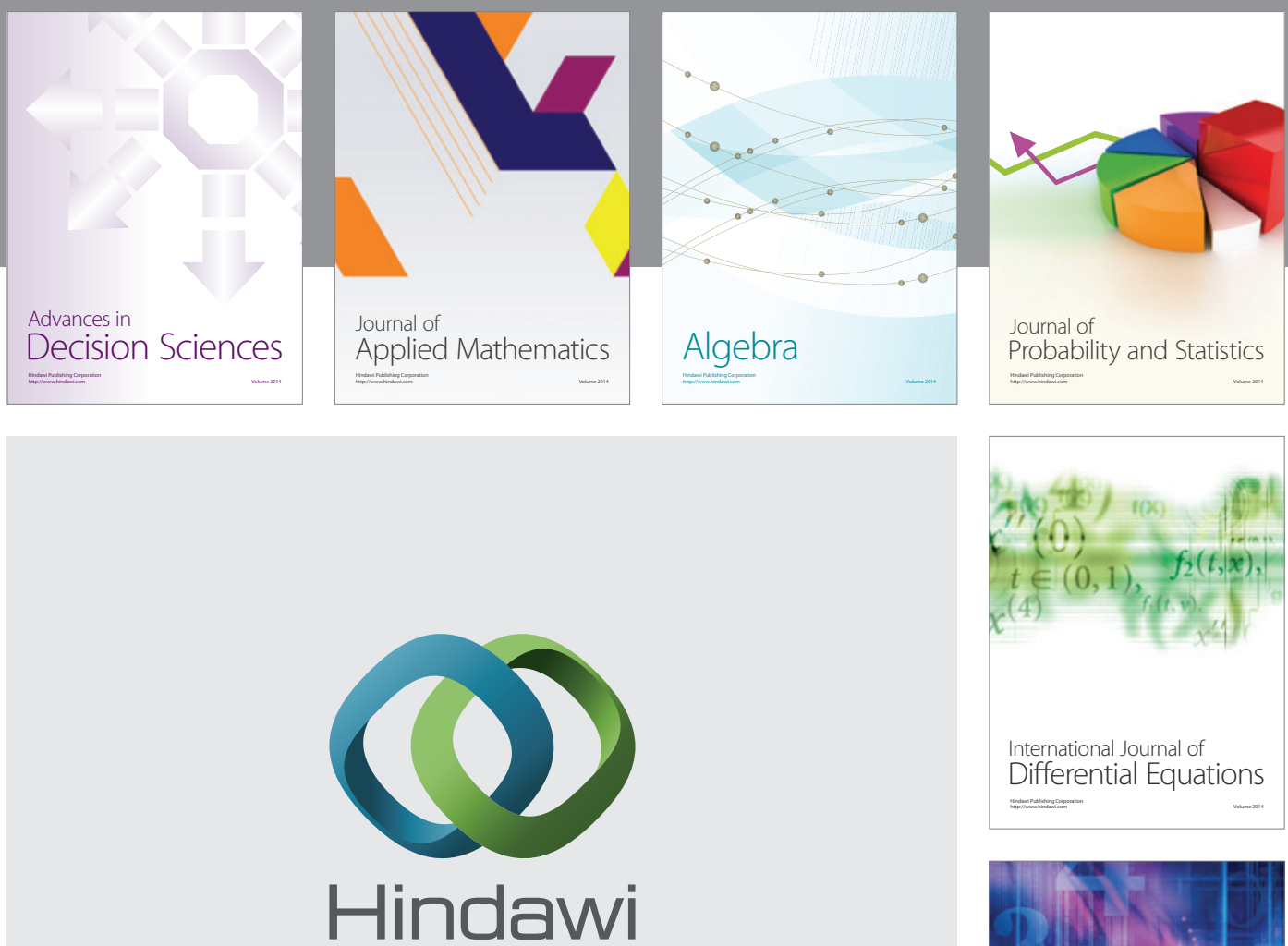

Submit your manuscripts at http://www.hindawi.com
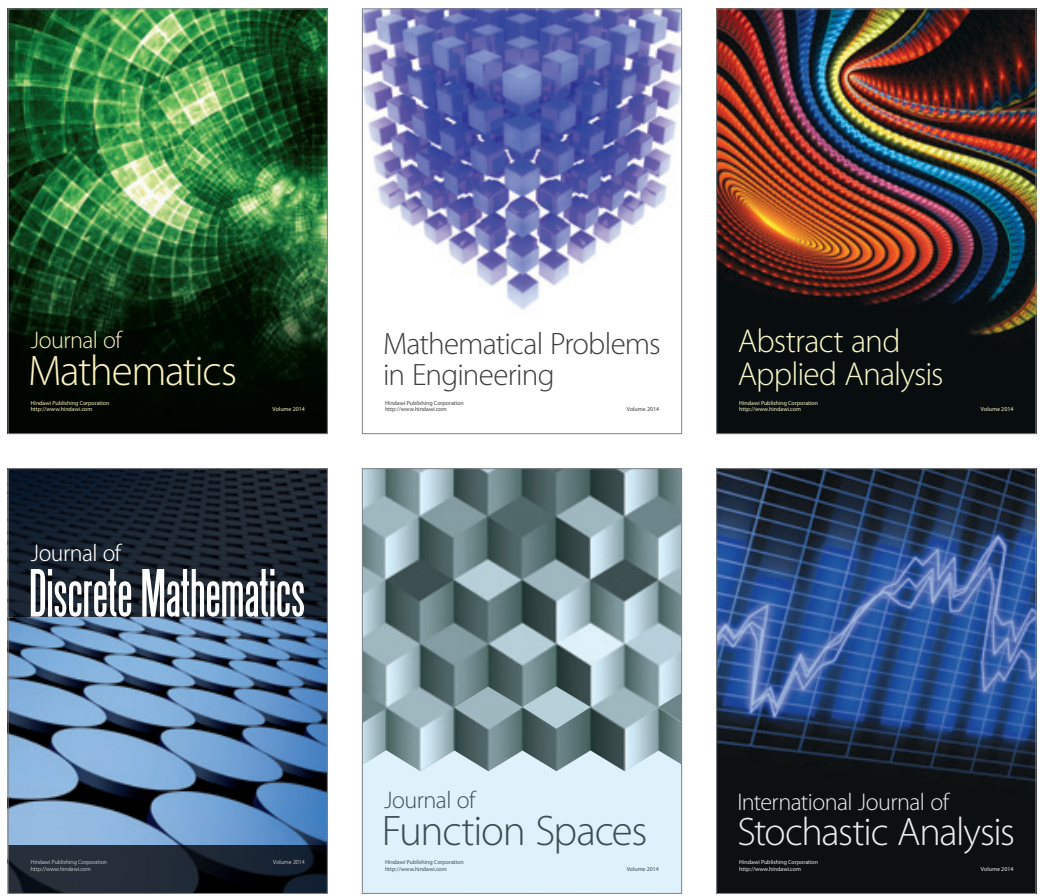

Journal of

Function Spaces

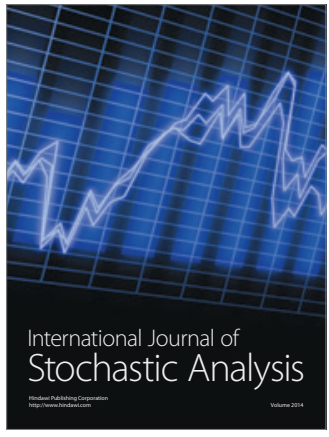

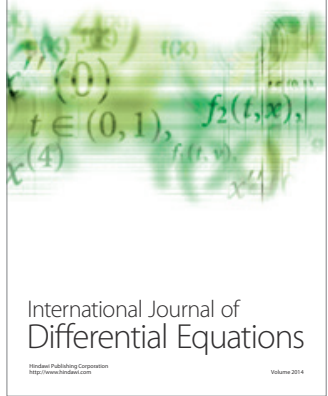
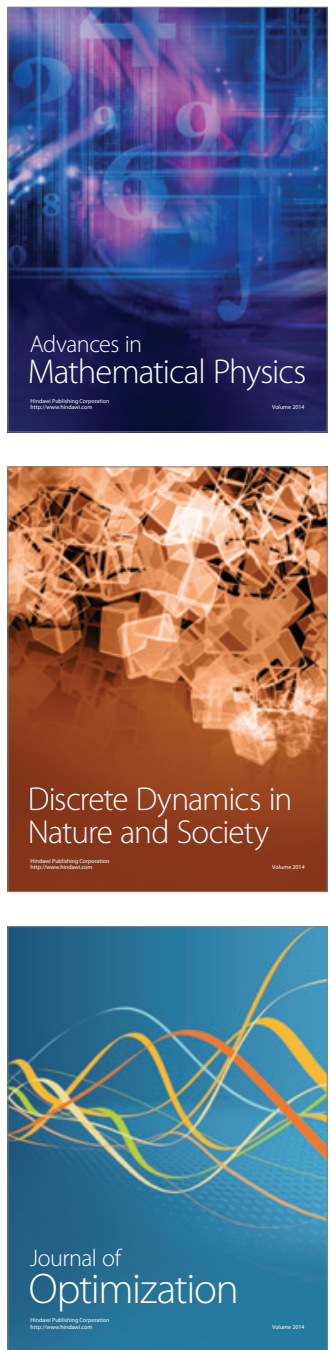